\title{
Multi-factor model and simulation of social cohesion and its effect on evacuation
}

\author{
Carole Adam \\ Univ. Grenoble-Alpes \\ Carole.Adam@imag.fr
}

\author{
Julie Dugdale \\ Univ. Grenoble-Alpes \\ Julie.Dugdale@imag.fr
}

\author{
Catherine Garbay \\ CNRS \\ Catherine.Garbay@imag.fr
}

\begin{abstract}
In this paper we propose an analysis of social cohesion in terms of 3 factors: emotions, social norms, and mutual knowledge. These factors have previously been analysed separately in terms of Beliefs, Desires, Intentions (BDI) logics, but have not been merged into a unified model. The goal of this paper is to provide a unified agent-based model and describe its implementation in the GAMA simulation platform. The simulator is applied to an evacuation case study and different scenarios are run to evaluate the impact of the 3 factors on cohesion, as well as the effect of cohesion on evacuation. This paper describes first results and concludes with interesting future prospects.
\end{abstract}

\section{Introduction}

Understanding social cohesion and its dynamics is becoming increasingly important in the current political and social situation. The aim is to support its positive aspects, such as solidarity, and prevent its negative aspects, such as divided communities. Crisis situations are particularly appropriate for observing social cohesion, its dynamics and its impact. In such situations new and unexpected forms of cohesion can emerge, such as altruism and solidarity, or racism and a lack of communication and openness between groups. Studying the dynamic behaviour of social cohesion in crisis situations cannot easily or safely be undertaken in real life. Therefore computer simulation offers a viable and powerful alternative. There exist various approaches to computer simulation, among which the agent-based approach is particularly suitable for modelling human behaviour at the micro level [1]. Agents are autonomous entities, capable of reactive and proactive behaviour depending on the context, and that can communicate with others. In these respects they are therefore well adapted to represent humans. As Bonabeau notes [2], agent based systems are able to capture emergent phenomena that are representative of complex adaptive systems, and provide a natural, realistic description of these systems. Finally, they are flexible, allowing us to study social systems at different levels of abstraction by varying the complexity of our agents or by aggregating agents into subgroups.

Modelling and simulating social cohesion has received very little attention in the literature. In this paper we introduce a first approach to this issue. We argue that there are three main factors that can influence cohesion and its dynamics: emotions, mutual knowledge, and norms (institutional rules or social conventions). Our contribution is to focus on the interplay between these three factors, how they evolve through environmental and social dynamics, and how they mediate an entity's individual beliefs, desires and intentions. Our analysis is grounded on a set of properties (autonomy vs dependence, altruism vs obedience, justice vs injustice) that show these complex relationships. A key result is to understand how these factors influence the efficacy and speed of evacuation.

The structure of the paper is as follows. Section 2 discusses the three main factors contributing to social cohesion, from a theoretical perspective and with a particular focus on their interaction. Section 3 then describes our agent based model of human behaviour including these factors. The presentation is anchored in an evacuation case study. This model is implemented in the GAMA platform and applied to practical scenarios, which are described in Section 4 along with the results of our experiments. Section 5 concludes this article with a discussion on the relevance of the findings to social cohesion, and ideas for future work.

\section{Factors of cohesion}

\subsection{Emotions}

The influence of emotions on human reasoning is now well accepted and many computer models of emotional agents exist. These models are generally based on psychological theories of cognitive appraisal $[3,4]$, that describe emotions as resulting from an 
individual's evaluation of the situation. This consists of two distinct processes: appraisal and coping. Appraisal triggers emotions in response to the individual assessment of the situation. This explains why different individuals may exhibit different emotions in the same situation, since the situation is evaluated differently by different people. Coping is an attempt to handle these emotions. This may be trying to suppress negative emotions via various strategies. Rational strategies, such as planning or action, may focus on the problem if it is controllable. Alternatively, strategies may focus on the emotion. These strategies may be more or less irrational and aim to modify the individual's mental attitudes with respect to the problem. Examples may be finding the silver lining, denying that the problem exists and ignoring clues, or taking one's mind off the problem.

Emotions seen as reasoning heuristics are even more relevant to study in crisis situations where decisions must be made quickly and with incomplete information. Research has shown that reasoning in crises is influenced by cognitive biases and affective heuristics [5]; these biases have been highlighted in natural disasters such as bushfires [6].

Other works show the importance of social attachment in evacuation after disasters such as earthquakes [7]. Attachment to a target (in a wide sense: object, place, person, group, etc) [8] is the source of emotions, for example, worry about one's family. Attachment is also the result of shared emotions, for example attachment to a familiar place that is linked with good memories, or to a person who has shared strong emotional experiences. Thus, strong cohesion can be observed in groups such as a fire brigade where members share the same emotions, understand and can empathise with each other. Research has shown that emotions rather than cognition determines team work in stressful situations [9].

Conversely, some emotions such as fear can hinder cohesion. Crisis managers are trained to suppress and not express these emotions in order not to break group cohesion. For instance firemen often use humour as a coping strategy, to regulate emotions, and as a bonding mechanism $[10,11,12,13]$.

\subsection{Social norms}

Cohesion is discussed in this section from an institutional and collaborative perspective, i.e an activity involving several organizations whose roles and missions are governed by different rules. It is therefore important to distinguish between the cohesion of individual activity, which depends on the individual's adherence to the rules governing its organization, and the cohesion of collective activity, which depends on the quality of coordination between actors.

In a crisis situation, the institutional rules differ according to the involved actors and the context. They are valid only in specific contexts, and are inherently incomplete because it is impossible to list all crisis situations and all possible responses. Their appropriation and mastery by the actors can be partial. Their application can be a source of error, because of the difficulties for the actors to perceive the global situation and its dynamics. In addition, at the inter-organizational level, one cannot assume that the set of rules is coherent and non-contradictory.

It is also important to distinguish between the prescribed activity and the real activity. The prescribed activity is described by a set of norms and whose cohesion depends on the coherence and comprehensiveness of this set. Whereas the real activity is that which is actually carried out by human actors and may not be in line with the norms, either through ignorance of the norms, or when the situation requires applying commonsense norms instead. Simulation plays a participative role and provides an evaluative feedback on the consequences of the modelled norms. It may lead to (i) an improvement in the completeness and coherence of the intra and inter-organizational sets of norms, and (ii) an improvement in the cohesion of the actors' behaviour towards these norms, i.e. to improve the appropriation and mutual awareness of these norms [14].

\subsection{Mutual knowledge}

Mutual knowledge is knowledge that interacting agents share and know they share [15]. It is also called 'common ground' because it is also an integral part of coordination actions and collaborative decision-making [16].

This notion can be analysed from the point of view of both victims and crisis managers. Previous work has shown that the first action of people in a crisis situation is not to search for shelter but to seek information [17], via traditional media (e.g. radio, TV), social media (Facebook, Twitter, SMS), or direct interactions with other individuals in the same situation. Information seeking aims at building situation awareness and understanding by the individual in order to prepare a coordinated response (e.g. collective flight decision). Seeking and sharing information through (physical or digital) social interactions allows the individual to improve their mutual knowledge of the situation, and strenghtens social links and cohesion.

From a theoretical point of view, the emergence 
of mutual awareness in a group can be explained as a percolation phenomenon [18] and is necessary for efficient cooperation and coordination in a collective task. However, the temporality of gaining mutual mutual knowlegde differs dramtically between everyday situations, where it can be gained over a long period of time, versus crisis situations, where victims typically only have brief interactions that strongly constrain the acquisition of mutual knowledge and make it imperfect.

Mutual knowledge can be established via several mechanisms [15]: direct knowledge created during shared experiences where individuals can obtain an informed judgement about what others know; interactional dynamics, when individuals meet the discussion is first focused on what is considered to be mutual knowledge [19]; and belonging to a category, which facilitates mutual knowledge. In crisis situations the usual social borders are broken and a new "victim" category emerges that reinforces social cohesion. Contrarily to non critical situations, where individuals are not likely to share their information [16], in crisis situations the need to understand the situation pushes them to share what they know. It has been shown that information sharing facilitates the emergence of mutual knowledge and can result in exceptional system robustness [20], which is a characteristic that emergency managers wish to obtain.

\subsection{Interactions between these 3 factors}

Our previous work leads us to look at the interaction between the gravity of crisis situations, emotions, mutual knowledge and norms. The gravity of a situation leads to the emergence of strong emotions and the obligation to obey specific norms. However, because of the tight time constraints it can also result in more effective communication since there is a conscious and concerted effort to inform others.

Emotions are linked to institutional norms in many ways. Norms can trigger certain emotions; they specify which emotions may or may not be expressed according to the context; they also provide a behavioral guide when the level of stress reduces the ability to make decisions. The moral or social emotions (e.g. shame and guilt, pride, admiration, etc.) felt towards a group come from respecting or violating the moral and cultural values shared by the group. These emotions can influence group cohesion by pushing to respect the norms or trying to repair the violations (repair actions, excuses) so that individuals can continue to be accepted in the group. The need to respect the norms can be inconsistent with individual goals and lead to dilemmas, such as choosing between a moral action or a selfish one.
According to von Scheve [21], the relationship between emotions and social norms, more precisely the place of emotions in normative multi-agent systems, have not yet been intensively studied, despite some pioneering work. Nevertheless it has been possible to show how emotion is essential in maintaining social norms, and in particular how sanctions may be imposed when there is non-compliance with norms. Furthermore, social norms, which are sources of emotion, can determine the processes of coping and regulating emotions.

Cairo [22] focuses on the articulation between institutional (rather formal) norms and "friendliness" (rather implicit) norms and on the impact of violating these two types of norms. He notes that friendliness appears rather as a "positive" norm, but also has a negative side when it intervenes to ground relations of power. He emphasizes the importance of friendliness to improve communication, increase the effectiveness of cooperation by reducing conflicts, and strengthen social cohesion by highlighting its values. Friendliness is brought into play through the expression of feelings, such as praising and encouraging people who conform to norms, and expressing resentment and anger towards those who do not. This is both a condition of social interaction and a valuable instrument for the internal regulation of social systems.

Emotions are linked to mutual knowledge in several ways. Beliefs about others can trigger positive emotions (e.g. empathy, compassion) or negative ones (e.g. resentment, jealousy, envy, etc.). Also through a process of reverse appraisal, the emotions expressed by others can be analyzed to build knowledge about the mental attitudes that may have caused that emotion. Finally, mutual knowledge favors the understanding of the emotions of the other agents and thus the cohesion of the group. The term emotional knowledge has been introduced by Shaver et al. [23] to describe sharing and describing emotional reactions to past or present events. This mutual emotional knowledge advantageously increases the efficiency of the social group [24]. In crisis situations, this can lead to groups of victims coordinating their response or evacuation actions.

Knowledge and understanding of dynamic situations are vital for deciding how to act and which normative rules to apply. Artefacts, such as social media, also play a role in supporting mutual awareness since they allow people to understand other peoples actions, possibly recognise their intentions, and to comprehend the emotions evoked by the situation. Social norms are in fact mutual knowledege that is shared by the collective. Mutual awareness of norms is an important pre-requisite, but is not limited to the 
sharing of formal knowledge, involving the sharing of beliefs and intentions over the situations encountered. Rauwolf et al. [25] discussed the importance of the notion of "normative awareness", emphasizing the need to distinguish it from the more common notion of "situation awareness". They propose three levels: perception, comprehension, and ability to anticipate the impact of norms of others. According to them it is the third level, that is to say the awareness that other agents are guided by their own beliefs and intentions, which is the most important to consider. The absence of studies and models on sharing intentionality leads to the simplifying assumption, which is present in most works, of a general awareness of all norms.

Emotions, norms, and mutual knowledge have already been studied independently in the scope of group behaviours, but they have not been combined in order to explain the dynamics of social cohesion. The goal of this work is to combine these factors in a unified agent model that shows their impact on the dynamics of beliefs and decisions of the agent at the micro scale, and on the effect of group cohesion. The following section describes the proposed unified model.

\section{Agent-based model of cohesion}

\subsection{Case study}

The case study concerns a situation of people evacuating from a large room in a building, for example a shopping mall. In order to practically demonstrate how the three factors identified above contribute to the emergence of social cohesion, the case study has been manipulated so that people can belong to one of two different social groups. Furthermore the room has a number of exits, some allowing only agents from one or the other social group to pass through. The number of exits can be parameterised, so that injust conditions can be created and studied. Not all of the agents know the location of exits, so they do not have mutual knowledge. However, as the simulation proceeds they can share their knowledge.

The environment is a large square room (in future it can be made more realistic by adding walls and furniture, etc, but it was not considered necessary at this point). The room contains an alarm system (located at the center of the room) that can be switched on or off, and a number of exits of two different types (square and circle). It is populated with a number of agents, representing humans, that belong to two different social groups (denoted by circles and squares). Their model is detailed below,

\subsection{Human agents attributes}

The human agents in the model have the following main psychological attributes: level of obedience to norms, i.e. how likely they are to respect the norms they are subject to; level of altruism, i.e. how likely they are to help other agents around them, for instance by providing information or guidance; level of leadership, i.e. how likely they are to explore for exits proactively versus just following other agents; risk aversion; and emotional sensitivity. Human agents also have various physical attributes such as a level of energy (which is depleted as they move around, and can be refilled when someone helps them), a list of known exits, and a radius of perception (how far they can see around them).

\subsection{Agents BDI model}

The BDI architecture based on formal BDI logics [26] is widely used in agent-oriented programming [27] and is starting to gain interest in social simulation in order to design more realistic agents [28], in particular when extended with emotions [29, 30]. Agents with this architecture have different types of mental attitudes: beliefs (what they know about the world); desires (how they would like the world to be, the desires may possibly be inconsistent or infeasible); intentions (these are selected feasible, consistent desires that the agent is committed to achieve with some degree of persistence). They might also have norms or ideals (which are externally imposed descriptions of how things should be). Finally they have a library of plans (recipes to reach their intentions).

The reasoning of BDI agents is organised in the following phases: perception of their environment to update their mental attitudes, and infer new mental attitudes from the existing ones based on a set of rules; decision: selecting an intention from the agents desires (usually the highest priority feasible desire is selected), and selecting a plan to achieve this intention in the current context; action: performing the first action of the current plan. Below we detail the specific features of the BDI agents in our model.

3.3.1. Predicates Logical predicates describe the agents world and represent mental attitudes. The necessary predicates, a sort of ontology of the domain of application, for our case study is shown in Table 1.

3.3.2. Perceptions, new beliefs The human agents can perceive the world around them, including other 


\begin{tabular}{|c|c|c|}
\hline shopping & None & the agent is currently busy shopping in the mall \\
\hline danger & Starting cycle & there is a danger (signalled by the alarm) \\
\hline injustice & Ratio of circle/square exits & the situation is injust (more exits for the others than self) \\
\hline at_exit & Which exit & the agent is at an exit \\
\hline know_exits & Which exits & the agent knows an exit (initial or after exploring) \\
\hline $\begin{array}{c}\text { circle_exit } \\
\text { square_exit }\end{array}$ & None & the agent can use circle exits \\
the agent can use square exits
\end{tabular}

Table 1. Domain ontology: predicates, values, meaning

agents. Their observations result in new beliefs, desires, social relationships, and emotions.

- Perception of the alarm being switched on adds a belief that there is danger, and removes the desire to keep shopping.

- Perceiving an exit within the agent's perception radius adds beliefs that the agent knows an exit, and that the agent is located at an exit.

- When the agent knows of more exits for the other group than for its own, it adds a belief that the situation is injust (this belief can be mistaken since it is based on the agent's incomplete perception of its environment: there could be more exits that it does not know about yet).

- Perceiving an exit at the immediate proximity adds a desire to exit, whose strength depends on the type of exit: if it is the correct exit, the priority is maximal; if it is an incorrect exit, the priority is moderated by the level of subjective danger (the higher the danger, the more chance to exit) and the level of obedience (the more obedient, the less chance of using the wrong exit)

- Perceiving another agent in perception radius creates a social link with them: if they are heading to an exit then trust towards them increases; if they are helping someone, then liking towards them increases; if they are in the same social group, solidarity increases.

- Perceiving an agent who is stopped, lost, out of energy, or waiting for a leader, adds a belief that they need help and a desire to help, with priority based on the agent's level of altruism.

- Perceiving an agent helping another agent (whether they provide energy or information about exits) creates a new belief about that helping action, storing who helped who.
Concerning the above perceptions, we assume that agents can access the other agents' current plan. This is a slight simplification, but it can be justified by the fact that observable actions usually suggest what people are doing: people heading straight to a known exit move faster and more confidently than people who are exploring around; people who are helping others usually slow down or stop next to them; etc.

3.3.3. Triggering emotions The emotions triggered in our agents are based on their definitions by Ortony, Clore and Collins [4] and on their logical formalisation by Adam [29].

- Hearing the alarm triggers fear of the potential danger, with an intensity based on the agent's risk aversion level. Fear intensity then increases with time as long as the alarm keeps ringing, making the potential danger more imminent.

- When the alarm stops, fear is suppressed immediately.

- Perceiving an agent needing help, needing information, or waiting for a leader, triggers compassion for that agent, with an intensity based on the agent's altruism and liking for the target agent.

- Perceiving an agent from a group that has more (known) exits than the other group triggers jealousy in the agents from that other group.

- Perceiving an agent helping someone else triggers admiration; if they are helping oneself it triggers gratitude.

3.3.4. Rules, new desires Rules describe logical links allowing the agents to infer new mental attitudes 
from the ones they already have and the ones they deduce from their observations above. In particular, these rules can activate contextual desires that appear only under some conditions. For instance we have the rules below:

- When the alarm is ringing and the agent knows of at least one exit, it infers the desire to head to the exit, with a priority based on its subjective evaluation of danger.

- When the alarm is ringing but the agent does not know any exits, it infers the desire to find exits, with a priority proportional to its level of leadership

- When the alarm is on and the agents knows no exit but knows potential leaders, it infers the desire to follow, with a strength inversely proportional to its leadership

- When the alarm is on, the agents knows no exit and no potential leader, it infers the desire to find a leader, with a strength inversely proportional to its leadership.

These situations are not mutually exclusive, so each agent might infer a number of different and possibly contradictory desires. For instance an agent knowing no exit might desire to find one itself (priority proportional to its leadership) and at the same time desire finding and following a leader towards an exit (priority inversely proportional to its leadership). The agents therefore need to rank their desires in order of priority, and select the one with the highest priority to make it their intention. In the previous example, an agent with high leadership will favor exploration for exits, while an agent with low leadership will prefer to find someone to follow. Contrary to desires, intentions have to be feasible, consistent, and persistent. Once selected, the agent commits to pursue its current intention until it is either reached, or deemed unfeasible (due to a change of context). Intentions lead to action because of the plan library.

3.3.5. Plans Our agents have a library of plans available to achieve their intentions in different contextual conditions.

- Intention to go shopping: there is only one plan that consists of wandering around in the shopping mall;

- Intention to be at the exit: there is only one plan, which is applicable when an exit is known, and which consists in heading straight to that exit;
- Intention to know an exit: two plans are applicable when an exit is not yet known. The first one is an organised exploration where the agent aims for a wall and then follows the wall until it finds an exit. The second one is a more disorganised exploration where the agent moves around randomly until it finds an exit by chance; it is selected if the first one fails for any reason;

- Intention to follow towards an exit: three plans are applicable. If a known leader is within reach, the first plan deals with following them. If no known leader is visible, but other agents are around, the second plan chooses the best one (either the closest, or fastest, or with highest trust / liking / altruism) and follows them. If no potential leader is within reach, the third plan allows the agent to follow the general direction of others around it;

- Intention to find a leader: there is a single plan that lets the agent wait until a leader comes past (its perception will trigger the belief that the agent now has a leader, which will finish the intention and plan to wait);

- Intention to exit: two plans are available when the agent is within reach of a door. The first plan is applicable if the exit is the correct one (i.e. allowed by the norms), and lets the agent use that door. The second plan is applicable if the exit is wrong but the agent believes that it is an emergency, or is not very obedient. If neither of these 2 plans is applicable (e.g. the agent is at the wrong door and exiting is not yet that urgent), the intention is unfeasible, dropped, and another desire comes forth (e.g. exploring to find more exits).

At each time step, the agent either continues executing its current plan, or selects a new one, or selects a new intention. Indeed, if a plan fails for some reason the agent will try another plan available for the same intention; if no plan is feasible for the current intention, it is deemed unfeasible, dropped, and another one is selected. Having backup plans for each intention ensures robust behaviour of the agents, making their behaviours more like real humans.

In the next section we describe how this model was implemented, and the different experiments that were conducted to evaluate cohesion, its links with the 3 investigated factors (emotions, norms, mutual knowledge), and its impact on evacuation. 


\section{Implementation and experiments}

\subsection{Implementation}

GAMA platform The model has been implemented and simulation have been run using the GAMA platform. GAMA is an open-source agent-based modelling and simulation platform, offering easy integration of GIS data into the models. It has recently been extended with a BDI architecture for the agents [31] as well as social and emotional features [32]. Agents with this architecture have their behaviour modelled in terms of beliefs, desires, intentions, norms. Emotions are triggered based on the agent's mental attitudes, they decay over time and are contagious. Agents can also create and maintain social relationships with other agents. GAMA is therefore well adapted to implement our BDI model of the three factors of cohesion.

User interface In the interface, the user can set the values of a number of parameters of the simulation, such as the density of people in the room (betwen 20 and 200 people), or the initial percentage of people that know an exit at the start of the simulation. The user can choose the minimum and maximum values of the different psychological attributes (altruism, leadership, and obedience to norms) in order to constrain the profile of the population. They can also use some actions to modify the simulation, in particular start and stop the alarm. They can also obtain feedback as the simulation is running though a console. In particular at each cycle information is provided about each agent, their current intention, plan, and level of energy. The main displays also show agents with a different colour based on their current plan. Other displays show emotions and their targets, and leader-follower groups (see Figure 1). When the simulation stops, statistics are provided about the total duration, percentage of agents evacuated, and if the norms were respected. By manipulating these parameters, we explored various scenarios to analyse the separated and combined influence of the 3 factors of social cohesion, and the impact of cohesion on evacuation.

\subsection{Measuring cohesion}

There is no consensus on the definition and measurement of cohesion. Based on our theoretical analysis above, we have devised several possible measures or indicators of cohesion, detailed here.

- Geographical measure: we used a DBscan algorithm [33] to create clusters of agents based on geographic proximity, which reflects groups of

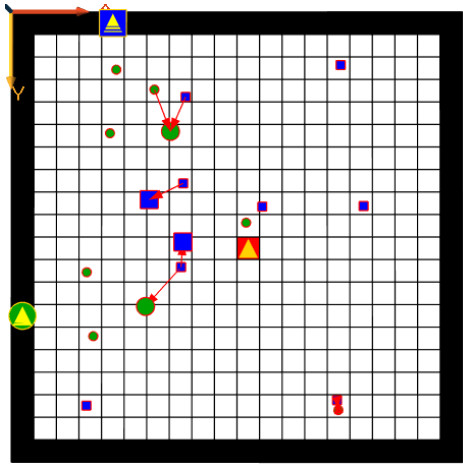

Figure 1. Display of the leader-follower groups emerging during evacuation.

agents following the same leader (see Figure 2). DBscan is a popular density-based clustering algorithm that when given a set of points in an environment it groups together points that are closely packed together (close neighbours). It also marks remote points that are alone in low-density areas (these are points whose nearest neighbours are too far away). We measured various properties of the clusters as a first indication of cohesion: number of groups, average size of groups, average lifetime of groups, and average time spent alone by the lonely agents.

- Emotional measure: we implemented a display in the simulator to show the agents, their social group (circle or square shape) and their emotions in the form of coloured labeled arrows (green for positive emotions, orange for negative ones) pointing at the target of the emotion. This display visualises the emotions shared inside of a group versus the emotions towards agents outside of the group.

- Normative measure: at the end of the simulation (when all agents have evacuated or stopped moving), the number of agents who have used the right exit, wrong exit, or no exit at all is measured. This gives an idea of cohesion in terms of adherence to norms: if all agents respect the norms, or all agents violate the norm, the population as a whole has more cohesion w.r.t. this norm than if behaviour is more varied.

- Informational measure: during the simulation, we measure the number of agents knowing of zero, one, or more exits. This provides an idea of cohesion in terms of information sharing, since at first only some agents know the location of exits. 


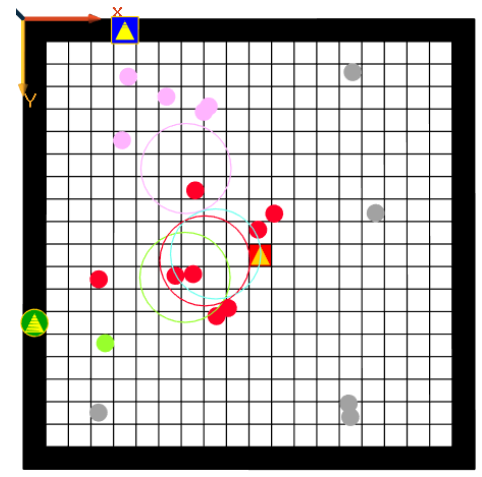

Figure 2. DBScan, 3 clusters (pink, green and red groups), 5 lonely agents (in grey).

\subsection{Scenario 1: autonomy vs dependence}

In a first scenario, we compared two extreme situations with respect to the initial knowledge of the agents, on a population of 20 agents:

- Situation 1: all agents know the location of the exit and have a high leadership. In this case, all agents are autonomous and immediately head to their known exit. No agent needs help, and no cohesion emerges.

- Situation 2: no agent knows the location of the exit. In that case, they explore the room to find an exit, some agents follow others. Since they lack information, they depend on others who might have more information than themselves to provide information or guidance. In this case cohesion emerges by need.

Table 2 shows the results in terms of geographical cohesion (number of groups and average size) and impact on evacuation (time and completeness). This suggests that with complete knowledge (which in our case study is limited to knowledge of the exits), the agents do not need each other and cohesion does not emerge.

\begin{tabular}{|c|c|c|c|c|}
\hline S & Evac. time & Evac. \% & Avg size & Nb groups \\
\hline S1 & 32 & 100 & 1 & 20 \\
\hline S2 & 106 & 90 & 3 & 8 \\
\hline
\end{tabular}

Table 2. Results of experiment 1: evacuation time and percentage, average size and number of groups, in both situations

\subsection{Scenario 2: obedience vs altruism}

In a second scenario we manipulated the parameters of obedience and altruism of the agents:
- Situation 1: the agents are very altruistic. They help each other and form more groups, guiding others to the exits. Since they do not consider social groups, they might guide someone to the wrong exit. As a result, many agents use the wrong exit, but very few agents, if any, are left behind.

- Situation 2: the agents are not altruistic but very obedient to norms. They do not stop to guide others. Agents who find a wrong exit first will refuse to use it due to their high obedience, so they will keep exporing to find a correct exit. As a result, the evacuation might be slower, with agents left behind, but most agents use the right exit (unless they feel it is too dangerous to obey the norm).

Table 3 shows the results of this experiment with populations of 20 agents.

\begin{tabular}{|c|c|c|c|c|}
\hline S & Correct & Wrong & Not exited & Time \\
\hline S1 & 15 & 2 & 3 & 127 \\
\hline S2 & 10 & 9 & 1 & 79 \\
\hline
\end{tabular}

Table 3. Results of experiment 2: agents exiting through correct door, incorrect door, not exiting at all, and total time.

This scenario suggests that not all forms of cohesion are the same. On the one hand, altruism creates cohesion around leaders who guide others to exits and share information about the exits, via the triggering of positive emotions of compassion, gratitude or admiration. This is an inclusive form of cohesion, regardless of the agents' norms or social groups: no one is excluded, and bigger groups emerge. On the other hand, obedience creates cohesion in terms of respecting the same norms. It creates reproach and other negative emotions towards those who have different norms and values, those who disobey and are not punished, or those who are favoured by unfair norms. This is an exclusive form of cohesion which depends on having the same norms or belonging to the same social group: insiders are cohesive but outsiders are rejected.

Further scenarios could investigate with "altruistic norms", for instance a norm that one should help others, or at least some types of others (children, elderly, women, etc). This could show differences in helping behaviours induced by altruism versus the same behaviours induced by enforcing norms. We suspect that norm-enforced helping behaviours would work well to counteract egoistic behaviours in normal situations, but would be less resistant to panic in case of an emergency, 
where individual survival will preempt if the person is not truly altruistic.

\subsection{Scenario 3: positive vs negative emotions}

The simulator allows triggering and visualising different types of emotions in the agents (see Figure 3).

- Positive emotions: altruistic agents feel compassion towards agents perceived as needing help. Agents receiving help feel gratitude towards those who help them, and are then more likely to help in return. They also feel admiration towards agents who are witnessed helping others, and are then more likely to choose them as leaders.

- Negative emotions: agents feel reproach towards agents violating a norm (not helping others or using the wrong door), and anger towards agents who refuse to help themselves. Subsequently, they are less likely to follow or help these disliked agents.

Positive emotions therefore play a role in cementing cohesion, making groups of agents emerge and stick together. On the contrary, negative emotions separate different groups, by excluding the agents who are the target of these emotions. For instance lost or weak agents will feel gratitude or admiration towards an altruistic leader who helped them by providing information or guidance, and they will follow them as a result. On the other hand egoistic agents might be rejected by agents who feel reproach towards them.

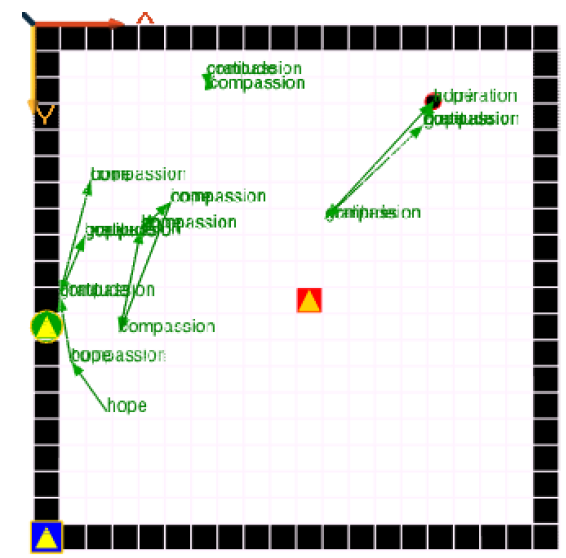

Figure 3. Displaying positive emotions inside 3 groups of agents.

\subsection{Scenario 4: justice vs injustice}

In the future we want to investigate scenarios where we create an unfair distribution of exits, with either more exits for one of the groups, or exits that are easier to locate. When perceiving such an unfair situation (less exits for one's own group), our agents feel jealousy towards any member of the social group having more exits, even though these agents are not directly responsible for this situation. This is often observed in the real world where quick categorisations and generalisations are made to judge people based on the group they belong to, in particular after crisis events. In such scenarios we hope to show the emergence of stronger cohesion in the discriminated group, by joining forces against a common enemy. We expect this to be a form of exclusive cohesion where foreign agents will be rejected for not sharing the same values.

\subsection{Impact of cohesion on evacuation}

Further experiments are needed to assess the impact of cohesion on evacuation in more detail. However, we can already see that cohesion helps some people evacuate that would not have been able to if left alone (see Figure 4). Indeed, some people are slower, shorter sighted, or ignore the location of exits. Having other agents who stop to help and guide them allows them reach the exit. On the contrary if they are unlucky and no one finds them (which can be the case with a lower density of agents in the room), they might panic or freeze and never evacuate. This suggests that evacuation might be slower but more complete with cohesive groups. Therefore, it is crucial that the alarm alerts the population well in advance so that there is more time to evacuate everybody.

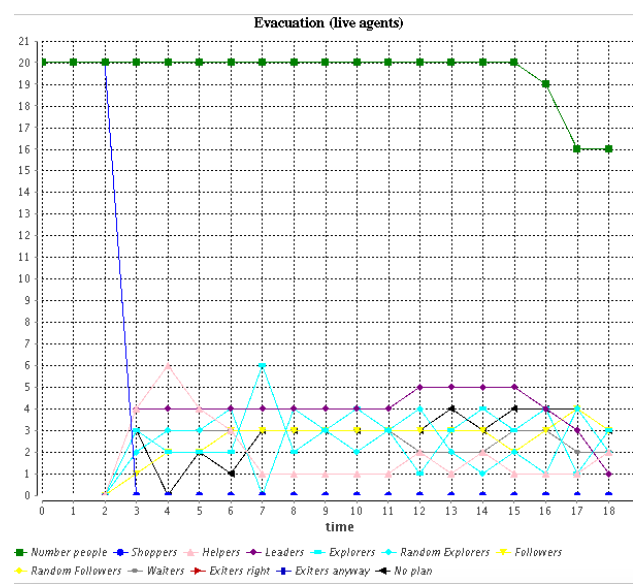

Figure 4. Evolution of distribution of plans in the agents. We can observe alarm starting at step 2, and first agent evacuating at step 15 


\section{Conclusion}

In this article we have shown that the dynamics of social cohesion result from the intertwining of several factors, namely emotions, mutual knowledge, and norms. We proposed a BDI agent model to integrate these 3 factors and implemented them in the GAMA platform. The interest of the BDI architecture is to offer a range of options to the agents where they can choose an intention among all their desires, and then choose one of the available plans to reach their intention. This allows us to simulate heterogeneous, non-predictable behaviour, where each agent has different priorities leading to different behaviours.

The experiments showed that cohesion may emerge in many different forms, with various bases and dynamics. Positive emotions, with behaviours driven towards the welfare of others have been shown to play a cementing role for an inclusive form of cohesion, relying on sharing. Conversely, unfair situations, with behaviours driven towards the obedience to shared norms, have been shown to create the emergence of exclusive forms of cohesion, relying on discrimination. The need for cohesion may also rely on the need for others (lack of information, lack of resources): no cohesion can emerge when the agents are fully autonomous.

How cohesion evolves and is analysed according to its positive or negative effects remains an open research field. Important work remains to be done to gain a deeper insight into the dynamics of cohesion, to account for the mutual dynamics of emotions, knowledge and norms, and to possibly integrate other factors of cohesion. Another important field of study would be to investigate how the search for and feeling of cohesion might bias individual behaviour, emotional response and obedience to norms. In future work we will develop a serious game simulating a crisis situation to test the influence of different aspects (quality of management plans and regulations, coordination of managers) on the emergence of cohesion.

\section{References}

[1] C. M. Macal and M. J. North, "Tutorial on agent-based modelling and simulation," Journal of simulation, vol. 4, no. 3, pp. 151-162, 2010

[2] E. Bonabeau, "Agent-based modeling: methods and techniques for simulating human systems," Proc. of the National Academy of Sciences, vol. 99, no. 3, 2002.

[3] R. S. Lazarus and S. Folkman, Stress, appraisal, and coping. New York: Springer, 1984.

[4] A. Ortony, G. Clore, and A. Collins, The cognitive structure of emotions. Cambridge Univ. Press, 1988.
[5] M. T. Kinateder, E. D. Kuligowski, P. A. Reneke, and R. D. Peacock, "A Review of Risk Perception in Building Fire Evacuation,” NIST Tech. Note 1840, NIST, 2014.

[6] M. Arnaud, C. Adam, and J. Dugdale, "The role of cognitive biases in reactions to bushfires," in Context'17, (Paris), June 2017.

[7] J. Bangate, J. Dugdale, C. Adam, and E. Beck, "A review on the influence of social attachment on human mobility during crises," in ISCRAM, 2017.

[8] A. R. Mawson, Mass panic and social attachment: the dynamics of human behavior. Ashgate, 2007.

[9] R. Nair, M. Tambe, and S. Marsella, "The role of emotions in multiagent teamwork," in Who needs emotions: the brain meets the robot (J.-M. Fellous and M. Arbib, eds.), pp. 311-329, Oxford Univ. Press, 2005.

[10] L. E. Alexander, When the bells go down: Resilience and vulnerability in firefighters. $\mathrm{PhD}$ thesis, University of Hertfordshire, 2016.

[11] C. Moran and E. Colless, "Positive reactions following emergency and disaster responses," Disaster Prevention and Management: An International Journal, vol. 4, no. 1, pp. 55-60, 1995.

[12] C. C. Moran, "Stress and emergency work experience: a non-linear relationship," Disaster Prevention and Management: An International Journal, vol. 7, no. 1, pp. 38-46, 1998.

[13] C. C. Moran, "Recruits predictions of positive reactions in diasaster and emergency work," Disaster Prevention and Management: An International Journal, vol. 8, no. 3, pp. 177-184, 1999.

[14] L. Thevin, J. Dugdale, O. Boissier, and C. Garbay, "Evaluating plans and human response using a normative multi-agent system," in ISCRAM, 2016.

[15] R. M. Krauss and S. R. Fussell, "Mutual knowledge and communicative effectiveness," in Intellectual Teamwork: Social and Technical Bases of Collaborative Work. (J. Galegher, R. Kraut, and C. Egido, eds.), Hillsdale, NJ: Erlbaum, 1990.

[16] C. Cramton, "The mutual knowledge problem and its consequences for dispersed collaboration," Organization Science, vol. 12, no. 3, pp. 346-371, 2001.

[17] J. Averill, D. Mileti, R. Peacock, E. Kuligowski, N. Groner, G. Proulx, P. Reneke, and H. Nelson, "Occupant behavior, egress, and emergency communications, federal building and fire safety investigation of the world trade center disaster," NCSTA report, NIST, 2005.

[18] J. Dugdale, N. Bellamine, F. Zouai, and B. Pavard, "Coupling agent based simulation with dynamic networks analysis to study the emergence of mutual knowledge as a percolation phenomenon.," J. Systems Science \& Complexity, vol. 29, no. 5, 2016.

[19] G. Stasser and W. Titus, "Effects of information load and percentage of shared information on the dissemination of unshared information during group discussion," $J$. Personality and Social Psychology, vol. 53, no. 1, 1987.

[20] B. Pavard, J. Dugdale, N. B.-B. Saoud, S. Darcy, and P. Salembier, "Underlying concepts in robustness and resilience and their use in designing socio-technical systems," in Resilience Engineering Perspectives, Remaining Sensitive to the Possibility of Failure (C. P. Nemeth, ed.), Ashgate, 2008. 
[21] C. von Scheve, D. Moldt, J. Fix, and R. von Luede, "My agents love to conform: Norms and emotion in the micro-macro link," Comput Math Organiz Theory, vol. 12, pp. 81-100, 2006.

[22] P. Caire, "A normative multi-agent systems approach to the use of conviviality for digital cities," in COIN, vol. III, pp. 245-260, 2007.

[23] P. Shaver, J. Schwartz, D. Kirson, and C. O'connor, "Emotion knowledge: further exploration of a prototype approach," Journal of personality and social psychology, vol. 52, no. 6, p. 1061, 1987.

[24] S. Rhee, "Shared emotions and group effectiveness: the role of broadening-and-building interactions," Academy of management Proceedings, vol. 2006, no. 1, 2006.

[25] P. Rauwolf, T. Balke, and M. De Vos, "Modelling normative awareness: First considerations," in Social Coord.: Principles, Artefacts \& Theories, 2013.

[26] A. S. Rao and M. P. Georgeff, "Modeling rational agents within a BDI-architecture," in 2nd Int. Conference on Principles of Knowledge Representation and Reasoning (J. A. Allen, R. Fikes, and E. Sandewall, eds.), pp. 473-484, Morgan Kaufmann, 1991.

[27] L. Padgham and M. M. Winikoff, Developing intelligent agent systems: a practical guide. Wiley, 2004.

[28] C. Adam and B. Gaudou, "BDI Agents in social simulations: a survey," The Knowledge Engineering Review, vol. 31, no. 3, pp. 207-238, 2016.

[29] C. Adam, A. Herzig, and D. Longin, "A logical formalization of the OCC theory of emotions," Synthese, vol. 168, no. 2, pp. 201-248, 2009.

[30] M. Bourgais, P. Taillandier, L. Vercouter, and C. Adam, "Emotion modeling in social simulation: a survey," Journal of Artificial Societies and Social Simulation (JASSS), vol. 21, March 2018.

[31] P. Taillandier, M. Bourgais, P. Caillou, C. Adam, and B. Gaudou, "A situated BDI agent architecture for the gama modelling and simulation platform," in $M A B S$ @ AAMAS, MABS, 2016.

[32] M. Bourgais, P. Taillandier, and L. Vercouter, "An Agent Architecture Coupling Cognition and Emotions for Simulation of Complex Systems," in SSC, 2016.

[33] M. Ester, H.-P. Kriegel, J. Sander, X. Xu, et al., "A density-based algorithm for discovering clusters in large spatial databases with noise.," in $K D D$, pp. 226-231, AAAI, 1996. 